International Journal of Research in Advent Technology, Vol.7, No.4, April 2019

E-ISSN: 2321-9637

Available online at www.ijrat.org

\title{
Measures of Similarity between Vague Binary Soft Sets
}

\author{
Remya. P. B ${ }^{1}$, Dr.Francina Shalini. A ${ }^{2}$ \\ Ph.D Research Scholar ${ }^{1}$, Assistant Professor ${ }^{2}$ \\ Department of Mathematics, Nirmala College for Women, Coimbatore, Tamil Nadu \\ krish3thulasi@gmail.com ${ }^{1}$,francshalu@gmail.com ${ }^{2}$
}

\begin{abstract}
Vague sets are one of the extensions of fuzzy sets. Vague binary soft set is one hybrid structure developed for dealing complicated situations with uncertainties with two universes. Similarity measures have a major role in application field of set theory. In this paper a similarity measure and weighted similarity measure is developed for measuring the degree of similarity between vague binary soft sets.
\end{abstract}

Keywords - vague binary soft set; similarity measure ; weighted similarity measure

Notations - In this paper, VBSS denotes vague binary soft set. $\mathrm{M}(\ddot{\mathrm{F}}, \mathrm{E}),(\ddot{\mathrm{G}}, \mathrm{E})), \mathrm{W}(\ddot{\mathrm{F}}, \mathrm{E}),(\ddot{\mathrm{G}}, \mathrm{E}))$ is used to denote similarity measures and weighted similarity measures of vague binary soft sets.

\section{INTRODUCTION}

Georg Cantor's classical set theory was inadequate to handle with several real life situations due to it's hard nature. So it need some reformations in time as a result so many other theories comes out viz., fuzzy set theory, intuitionistic fuzzy set theory, rough set theory, interval mathematics, vague set theory, soft set theory, neutrosophic set theory etc. These theories have their own positives and negatives. To overcome the negatives and extracting the positives some new hybrid structures like fuzzy soft, soft fuzzy, intuitionistic fuzzy, rough neutrosophic, vague soft, neutrosophic soft etc arose. These theories are used in a great extent to practical problems consisting uncertainties and vagueness where hard set theory always found to fail. Also the researches involving them are moving forward in a rapid speed globally. This paper concentrates in a particular area called similarity measures of one hybrid structure vague binary soft sets, developed by Dr. Francina Shalini. A. and Remya.P. B [5] in 2018. Similarity measures can be used to measure how much two sets or patterns or images are alike. In other words they can tell 'How much fuzzy ? '- a fuzzy set is ! or ' How much vague ?'-a vague set is! etc. Similarity measures based on set theoretical approach, distance and matching function which satisfying some axiomatic conditions are well known. Entropy and distance measures can also furnish the same role as that of similarity measures. i.e., they are also some kind of measurement tools used in the above mentioned theories with uncertainties. So these three together can work remarkably and can produce several useful theorems and formulae in this area. Similarity measures and distances are duals since large distance show low degree of similarity and vice versa. So distance measures could be used to define similarity measures. Problem under consideration decides which kind of similarity measure to be chosen. Wide applications of this topic in pattern recognition, decision making medical diagnosis, signal detection, security verification systems etc attracted the attention of researchers, in a commenting manner to this area, nowadays.

Molodtsov [5] introduced soft set theory in 1999 to remove all the existing difficulties of traditional set theory. Free of restrictions in describing the parameter set made soft set theory more convenient and user friendly. In 2010 Athar Kharal [1] introduced distance measures and similarity measures for soft sets. 'Majumdar' and 'Samanta' introduced similarity measure for soft sets based on distances using soft matrices. They [9] also introduced in 2011 similarity measures for fuzzy soft sets based on three different measures set-theoretical approach, matching function and distance. In 1995 Shyi-Ming Chen [12] proposed two similarity measures for measuring the degree of similarity between vague sets. In 2005, Jingli lu et al.,[7] presented a new similarity measure for vague sets. In 2006 , Faxin Zhao et al., [4] gave similarity measures for vague sets based on set theoretical method. Feng Sheng Xu [6] gave a new method on measures of similarity between vague sets in 2009 . Qinrong Feng and Weinan Zheng [11] gave new similarity measures for fuzzy soft sets in 2013 based on different distance measures viz., normalized hamming distance, normalized Euclidean, normalized hausdorff, hamming-hausdorff, chebyshev etc. They also gave one application based on distances. In 2014 Zhicai Liu et al., [14] introduced so many similarity measures for fuzzy soft sets and pointed out draw backs of some of them . In 2015 Wenyi Zeng, Yibin Zhao and Yundong $\mathrm{Gu}$ [13] proposed similarity measure for vague sets based on implication functions. Chang Wang and Anjing Qu [2] proposed axiomatic definition soft entropy, similarity measure and distance measure for vague soft sets in 2013. They also put forward some formulas to calculate them and some relative theorems. In 2014 Dan Hu, Zhiyong Hong and Yong Wang [3] proposed a new approach to 


\section{Available online at www.ijrat.org}

entropy and similarity measure of vague soft sets. In 2017 Manash Jyoti Borah and Bipan Hazarika [8] gave some applications of soft sets. They gave some new notions like exact fuzzy soft points, pointwise partial similarity etc. In this paper similarity measures and weighted similarity measures are developed for vague binary soft sets.

\section{PRELIMINARIES}

\section{Definition 2.1. [2, 3, 10] (Vague soft set)}

Let $\mathrm{U}$ be an initial universal set, $\mathrm{V}(\mathrm{U})$ be the power set of vague sets on $U$ and $E$ be the set of parameters with $\mathrm{A} \subseteq \mathrm{E}$. A pair $(\hat{F}, \mathrm{~A})$ is called a vague soft set over $\mathrm{U}$, where $\hat{F}$ is a mapping given by $\hat{F}: \mathrm{A} \rightarrow \mathrm{V}(\mathrm{U})$

\section{Definition 2.2. [5]}

Let $\mathrm{U}_{1}, \mathrm{U}_{2}$ be two initial universes which is common to a fixed set $A \subseteq E$ of parameters. Let $V\left(U_{1}\right)$ and $V\left(U_{2}\right)$ denote the power set of vague sets on $U_{1}, U_{2}$ respectively and $\mathrm{A} \subseteq \mathrm{E}$. A pair $(\ddot{F}, \mathrm{~A})$ is said to be a vague binary soft set over $\mathrm{U}_{1}, \mathrm{U}_{2}$ where $\ddot{F}$ is a mapping given by $\ddot{F}: \mathrm{A} \rightarrow \mathrm{V}\left(\mathrm{U}_{1}\right) \times \mathrm{V}\left(\mathrm{U}_{2}\right)$ and $(\ddot{F}, \mathrm{~A})=\{\mathrm{e} \in \mathrm{A} /(\mathrm{e}, \ddot{F}(\mathrm{e}))\}$

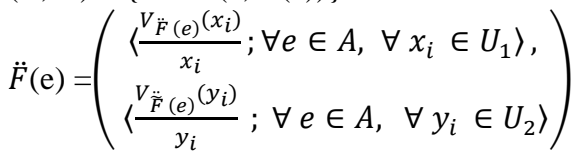

\section{Lemma 2.3. [10]}

Let $\left(F_{1}, E_{1}\right)$ and $\left(F_{2}, E_{2}\right)$ be two soft sets over the same finite universe $U$. Then the following conditions hold:
(1) $S\left(F_{1}, F_{2}\right)=S\left(F_{2}, F_{1}\right)$
(2) $0 \leq S\left(F_{1}, F_{2}\right) \leq 1$
(3) $S\left(F_{1}, F_{2}\right)=1$

Definition 2.4. [12]

$\mathrm{A}$ and $\mathrm{B}$ are two vague sets over the universe of discourse $\mathrm{U}=\left\{u_{1}, u_{2}, \ldots, u_{n}\right\}$.

$\mathrm{V}_{\mathrm{A}}\left(u_{i}\right)=\left[t_{A}\left(u_{i}\right), 1-f_{A}\left(u_{i}\right)\right]$ is the membership value of $u_{i}$ in vague set $\mathrm{A}$ and $\mathrm{V}_{\mathrm{B}}\left(u_{i}\right)=\left[t_{B}\left(u_{i}\right), 1-f_{B}\left(u_{i}\right)\right]$ is the membership value of $u_{i}$ in vague set $\mathrm{B}$.

Let $\mathrm{A}=\sum_{i=1}^{n}\left[\frac{\mathrm{t}_{\mathrm{A}}\left(u_{i}\right), 1-f_{A}\left(u_{i}\right)}{u_{i}}\right], \mathrm{B}=\sum_{i=1}^{n}\left[\frac{\mathrm{t}_{\mathrm{B}}\left(u_{i}\right), 1-f_{B}\left(u_{i}\right)}{u_{i}}\right]$.

Similarity between vague sets $A$ and $B$ can be obtained by the function,

$\mathrm{T}(\mathrm{A}, \mathrm{B})=\frac{1}{n} \sum_{i=1}^{n} M\left(\mathrm{~V}_{\mathrm{A}}\left(u_{i}\right), \mathrm{V}_{\mathrm{B}}\left(u_{i}\right)\right)$

$=\frac{1}{n} \sum_{i=1}^{n}\left(1-\left|\frac{S\left(V_{A}\left(u_{i}\right)\right)-S\left(V_{B}\left(u_{i}\right)\right)}{2}\right|\right) ; \mathrm{T}(\mathrm{A}, \mathrm{B}) \in[0,1]$.

Larger value of $\mathrm{T}(\mathrm{A}, \mathrm{B})$ indicates more similarity between the sets.

Definition 2.5. [3]

Let $\mathrm{M}: \operatorname{VSS}(\mathrm{U}) \times \operatorname{VSS}(\mathrm{U}) \rightarrow[0,1]$ be a mapping.

For $(\mathrm{F}, \mathrm{E}) \in \operatorname{VSS}(\mathrm{U})$ and $(\mathrm{G}, \mathrm{E}) \in \operatorname{VSS}(\mathrm{U}), \mathrm{M}((\mathrm{F}, \mathrm{E}),(\mathrm{G}, \mathrm{E}))$ is called the degree of similarity between $(F, E)$ and $(\mathrm{G}, \mathrm{E})$ if it satisfies the following conditions $\left(\mathrm{M}_{1}\right) \mathrm{M}((\mathrm{F}, \mathrm{E}),(\mathrm{G}, \mathrm{E}))=\mathrm{M}((\mathrm{G}, \mathrm{E}),(\mathrm{F}, \mathrm{E}))$

$\left(\mathrm{M}_{2}\right) \mathrm{M}((\mathrm{F}, \mathrm{E}),(\mathrm{G}, \mathrm{E})) \in[0,1]$

$\left(\mathrm{M}_{3}\right) \mathrm{M}((\mathrm{F}, \mathrm{E}),(\mathrm{G}, \mathrm{E}))=1 \Leftrightarrow(\mathrm{F}, \mathrm{E})=(\mathrm{G}, \mathrm{E})$

$\left(\mathrm{M}_{4}\right) \mathrm{M}((\mathrm{F}, \mathrm{E}),(\mathrm{G}, \mathrm{E}))=0 \Leftrightarrow$ for all $\mathrm{e} \in \mathrm{E}, \mathrm{x} \in \mathrm{U}$

$$
\begin{array}{r}
t_{F(e)}(\mathrm{x})=0, \quad f_{F(e)}(\mathrm{x})=1 ; t_{G(e)}(\mathrm{x})=1, \quad f_{G(e)}(\mathrm{x})=0 \\
\text { or } \\
t_{F(e)}(\mathrm{x})=1, f_{F(e)}(\mathrm{x})=0 ; t_{G(e)}(\mathrm{x})=0, f_{G(e)}(\mathrm{x})=1 \\
\left(\mathrm{M}_{5}\right)(\mathrm{F}, \mathrm{E}) \subseteq(\mathrm{G}, \mathrm{E}) \subseteq(\mathrm{P}, \mathrm{E}) \Rightarrow \mathrm{M}((\mathrm{F}, \mathrm{E}),(\mathrm{P}, \mathrm{E})) \leq \\
\min (\mathrm{M}((\mathrm{F}, \mathrm{E}),(\mathrm{G}, \mathrm{E})), \mathrm{M}((\mathrm{G}, \mathrm{E}),(\mathrm{P}, \mathrm{E}))
\end{array}
$$

\section{Definition 2.6. [2]}

Let $U=\left\{x_{1}, x_{2}, \ldots, x_{n}\right\}$ be the universal set of elements and $\mathrm{E}=\left\{\mathrm{e}_{1}, \mathrm{e}_{2}, \ldots, \mathrm{e}_{\mathrm{m}}\right\}$ be the universal set of parameters. Hence $(F, E)=\left\{F\left(e_{i}\right) / i=1,2, . ., m\right\}$ and $(G, E)=\left\{G\left(e_{i}\right) / i=1,2, \ldots, m\right\}$ are two families of vague soft sets. Define $\mathrm{M}((\mathrm{F}, \mathrm{E}),(\mathrm{G}, \mathrm{E}))$ as follows: $\mathrm{M}((\mathrm{F}, \mathrm{E}),(\mathrm{G}, \mathrm{E}))=\frac{\sum_{i=1}^{m} M_{i}((F, E),(G, E))}{m}$;

$\mathrm{M}_{\mathrm{i}}((\mathrm{F}, \mathrm{E}),(\mathrm{G}, \mathrm{E}))=$

$1-\frac{1}{4 n} \sum_{j=1}^{n}\left[\begin{array}{l}\left|S_{F\left(e_{i}\right)}\left(x_{j}\right)-S_{G\left(e_{i}\right)}\left(x_{j}\right)\right| \\ +\left|t_{F\left(e_{i}\right)}\left(x_{j}\right)-t_{G\left(e_{i}\right)}\left(x_{j}\right)\right| \\ +\left|f_{F\left(e_{i}\right)}\left(x_{j}\right)-f_{G\left(e_{i}\right)}\left(x_{j}\right)\right|\end{array}\right]$.

$S_{F\left(e_{i}\right)}\left(x_{j}\right)=t_{F\left(e_{i}\right)}\left(x_{j}\right)-f_{F\left(e_{i}\right)}\left(x_{j}\right)$ and

$S_{G\left(e_{i}\right)}\left(x_{j}\right)=t_{G\left(e_{i}\right)}\left(x_{j}\right)-f_{G\left(e_{i}\right)}\left(x_{j}\right)$ called core of $\mathrm{F}\left(e_{i}\right)$ and $\mathrm{G}\left(e_{i}\right)$ or degree of support of $\mathrm{F}\left(\mathrm{e}_{\mathrm{i}}\right)$ and $\mathrm{G}\left(\mathrm{e}_{\mathrm{i}}\right)$ respectively, $S_{F\left(e_{i}\right)}\left(x_{j}\right) \in[-1,1], S_{G\left(e_{i}\right)}\left(x_{j}\right) \in[-1,1]$ Then $\mathrm{M}((\mathrm{F}, \mathrm{E}),(\mathrm{G}, \mathrm{E}))$ is the similarity measure between two vague soft sets $(F, E)$ and $(G, E)$

Definition 2.7. [12]

Let $A$ and $B$ be two vague sets in the universe of discourse $\mathrm{U}=\left\{\mathrm{u}_{1}, \mathrm{u}_{2}, \ldots, \mathrm{u}_{\mathrm{n}}\right\}$.

$\mathrm{A}=\frac{\sum_{i=1}^{n}\left[\mathrm{t}_{\mathrm{A}}\left(\mathrm{u}_{\mathrm{i}}\right), 1-f_{A}\left(\mathrm{u}_{\mathrm{i}}\right)\right]}{u_{i}}, \mathrm{~B}=\frac{\sum_{i=1}^{n}\left[\mathrm{t}_{\mathrm{B}}\left(\mathrm{u}_{\mathrm{i}}\right), 1-f_{B}\left(\mathrm{u}_{\mathrm{i}}\right)\right]}{u_{i}}$, $1 \leq \mathrm{i} \leq \mathrm{n}$. Let $\mathrm{w}_{\mathrm{i}}$ be the weight of the element $\mathrm{u}_{\mathrm{i}}$ in the universe of discourse $U$, where $w_{i} \in[0,1]$ and $1 \leq \mathrm{i} \leq \mathrm{n}$. Using weighting function $\mathrm{W}$ similarity between the vague sets $A$ and $B$ can be evaluated using the formula below: $\mathrm{W}(\mathrm{A}, \mathrm{B})=$ $\frac{\sum_{i=1}^{n} w^{*} M\left(V_{A}\left(u_{i}\right), V_{B}\left(u_{i}\right)\right)}{\sum_{i=1}^{n} w_{i}}=\frac{\sum_{i=1}^{n} w_{i}^{*}\left(1-\left|\frac{S\left(V_{A}\left(u_{i}\right)\right)-S\left(V_{B}\left(u_{i}\right)\right)}{2}\right|\right)}{\sum_{i=1}^{n} w_{i}}$ where $\mathrm{W}(\mathrm{A}, \mathrm{B}) \in[0,1]$; Larger value of $\mathrm{W}(\mathrm{A}, \mathrm{B})$ indicates more similarity between vague sets $\mathrm{A}$ and $\mathrm{B}$

\section{SIMILARITY MEASURES FOR VBSS's}

In this section a similarity measure for vague binary soft sets is developed

\section{Definition 3.1.}

Let M:((VSS $\left.\left(\mathrm{U}_{1}\right) \times \operatorname{VSS}\left(\mathrm{U}_{2}\right)\right) \times\left(\left(\mathrm{VSS}\left(\mathrm{U}_{1}\right) \times \operatorname{VSS}\left(\mathrm{U}_{2}\right)\right)\right.$ $\rightarrow[0,1]$ be a mapping. Let $(\ddot{F}, \mathrm{~A})$ and $(\ddot{G}, \mathrm{~B})$ be two VBSS's such that, $(\ddot{F}, \mathrm{~A}) \in \operatorname{VSS}\left(\mathrm{U}_{1}\right) \times \operatorname{VSS}\left(\mathrm{U}_{2}\right)$ and $(\ddot{G}, \mathrm{~B}) \in \operatorname{VSS}\left(\mathrm{U}_{1}\right) \times \operatorname{VSS}\left(\mathrm{U}_{2}\right)$. Then $\mathrm{M}((\ddot{F}, \mathrm{~A}),(\ddot{G}, \mathrm{~B}))$ is called the similarity measure between vague binary soft sets if it satisfies the following conditions:
$\left(\mathrm{C}_{1}\right) \mathrm{M}((\ddot{F}, \mathrm{~A}),(\ddot{G}, \mathrm{~B}))=\mathrm{M}((\ddot{G}, \mathrm{~B}),(\ddot{F}, \mathrm{~A}))$
$\left(\mathrm{C}_{2}\right) \mathrm{M}((\ddot{F}, \mathrm{~A}),(\ddot{G}, \mathrm{~B})) \in[0,1]$
$\left(\mathrm{C}_{3}\right) \mathrm{M}((\ddot{F}, \mathrm{~A}),(\ddot{G}, \mathrm{~B}))=1 \Leftrightarrow(\ddot{F}, \mathrm{~A})=(\ddot{G}, \mathrm{~B})$ 


\section{Available online at www.ijrat.org}

$\left(\mathrm{C}_{4}\right) \mathrm{M}((\ddot{F}, \mathrm{~A}),(\ddot{G}, \mathrm{~B}))=0 \Leftrightarrow$

$\forall \mathrm{e}_{\mathrm{i}} \in(\mathrm{A} \cap \mathrm{B}), \forall \mathrm{x}_{\mathrm{j}} \in U_{1}$

$t_{\ddot{F}(e)}\left(x_{j}\right)=0, t_{\ddot{G}(e)}\left(x_{j}\right)=1$ or $t_{\ddot{F}(e)}\left(x_{j}\right)=1, t_{\ddot{G}(e)}\left(x_{j}\right)=0 \&$

$f_{\ddot{F}(e)}\left(x_{j}\right)=0, f_{\ddot{G}(e)}\left(x_{j}\right)=1$ or $f_{\ddot{F}(e)}\left(x_{j}\right)=1, f_{\ddot{G}(e)}\left(x_{j}\right)=0$

$\& \forall \mathrm{e}_{\mathrm{i}} \in(\mathrm{A} \cap \mathrm{B}), \forall \mathrm{y}_{\mathrm{k}} \in U_{2}$,

$t_{\ddot{F}(e)}\left(y_{k}\right)=0, t_{\ddot{G}(e)}\left(y_{k}\right)=1$ or $t_{\ddot{F}\left(e_{i}\right)}\left(y_{k}\right)=1, t_{\ddot{G}(e)}\left(y_{k}\right)=0 \&$

$f_{\ddot{F}(e)}\left(y_{k}\right)=0, f_{\ddot{G}(e)}\left(y_{k}\right)=1$ or $f_{\ddot{F}(e)}\left(y_{k}\right)=1, f_{\ddot{G}(e)}\left(y_{k}\right)=0$

$\left(\mathrm{C}_{5}\right)(\ddot{F}, A) \subseteq(\ddot{G}, B) \subseteq(\ddot{P}, C) \Rightarrow \mathrm{M}((\ddot{F}, \mathrm{~A}),(\ddot{P}, \mathrm{C}))$ $\min (\mathrm{M}((\ddot{F}, A),(\ddot{G}, B)), \mathrm{M}((\ddot{G}, B),(\ddot{P}, C)))$; where $(\ddot{P}, \mathrm{C}) \in \operatorname{VSS}\left(\mathrm{U}_{1}\right) \times \operatorname{VSS}\left(\mathrm{U}_{2}\right)$ and $\mathrm{A}, \mathrm{B}, \mathrm{C} \subseteq \mathrm{E}$

\section{Definition 3.2.}

(Similarity Measure between two VBSS's)

Let $U_{1}=\left\{\mathrm{x}_{1}, \mathrm{x}_{2}, \ldots, \mathrm{x}_{\mathrm{n}}\right\}, U_{2}=\left\{\mathrm{y}_{1}, \mathrm{y}_{2}, \ldots, \mathrm{y}_{\mathrm{p}}\right\}$ be the common universal set and $\mathrm{E}=\left\{\mathrm{e}_{1}, \mathrm{e}_{2}, \ldots, \mathrm{e}_{\mathrm{m}}\right\}$ be the set of parameters. Hence $(\ddot{F}, \mathrm{~A})=\left\{\ddot{F}_{e_{i}} / \mathrm{i}=1,2, \ldots, \mathrm{m}\right\}$ and $(\ddot{G}, \mathrm{~B})=\left\{\ddot{G}_{e_{i}} / \mathrm{i}=1,2, \ldots, \mathrm{m}\right\}$ are two families of VBSS's. Define M (( $\ddot{F}, \mathrm{~A}),(\ddot{G}, \mathrm{~B}))$ as follows :

$\mathrm{M}((\ddot{F}, \mathrm{~A}),(\ddot{G}, \mathrm{~B}))=\frac{\sum_{e_{i} \in(A \cap B)} M_{e_{i}}((\ddot{F}, \mathrm{~A}),(\ddot{G}, \mathrm{~B}))}{\operatorname{Card}(A \cup B)}$ where

$$
\begin{aligned}
& M_{e_{i}}((\ddot{F}, \mathrm{~A}),(\ddot{G}, \mathrm{~B}))= \\
& 1-\frac{1}{8 n} \sum_{j=1}^{n}\left[\begin{array}{l}
\left|S_{\ddot{F}\left(e_{i}\right)}\left(x_{j}\right)-S_{\ddot{G}\left(e_{i}\right)}\left(x_{j}\right)\right| \\
+\left|t_{\ddot{F}\left(e_{i}\right)}\left(x_{j}\right)-t_{\ddot{G}\left(e_{i}\right)}\left(x_{j}\right)\right| \\
+\left|f_{\ddot{F}\left(e_{i}\right)}\left(x_{j}\right)-f_{\ddot{G}\left(e_{i}\right)}\left(x_{j}\right)\right|
\end{array}\right] \\
& -\frac{1}{8 p} \sum_{k=1}^{p}\left[\begin{array}{l}
\left|S_{\ddot{F}\left(e_{i}\right)}\left(y_{k}\right)-S_{\ddot{G}\left(e_{i}\right)}\left(y_{k}\right)\right| \\
+\left|t_{\ddot{F}\left(e_{i}\right)}\left(y_{k}\right)-t_{\ddot{G}\left(e_{i}\right)}\left(y_{k}\right)\right| \\
+\left|f_{\ddot{F}\left(e_{i}\right)}\left(y_{k}\right)-f_{\ddot{G}\left(e_{i}\right)}\left(y_{k}\right)\right|
\end{array}\right]
\end{aligned}
$$

Now the following proof shows that the above defined similarity measure clearly satisfies the conditions given in Def 3.1. Hence Def 3.2 is clearly a similarity measure for VBSS's.

\section{Proof}

$\left(\mathrm{C}_{1}\right) M_{e_{i}}((\ddot{F}, \mathrm{~A}),(\ddot{G}, \mathrm{~B}))=$

$$
\begin{gathered}
1-\frac{1}{8 n} \sum_{j=1}^{n}\left[\begin{array}{l}
\left|S_{\ddot{F}\left(e_{i}\right)}\left(x_{j}\right)-S_{\ddot{G}\left(e_{i}\right)}\left(x_{j}\right)\right| \\
+\left|t_{\ddot{F}\left(e_{i}\right)}\left(x_{j}\right)-t_{\ddot{G}\left(e_{i}\right)}\left(x_{j}\right)\right| \\
+\left|f_{\ddot{F}\left(e_{i}\right)}\left(x_{j}\right)-f_{\ddot{G}\left(e_{i}\right)}\left(x_{j}\right)\right|
\end{array}\right] . \\
-\frac{1}{8 p} \sum_{k=1}^{p}\left[\begin{array}{c}
\left|S_{\ddot{F}\left(e_{i}\right)}\left(y_{k}\right)-S_{\ddot{G}\left(e_{i}\right)}\left(y_{k}\right)\right| \\
+\left|t_{\ddot{F}\left(e_{i}\right)}\left(y_{k}\right)-t_{\ddot{G}\left(e_{i}\right)}\left(y_{k}\right)\right| \\
+\left|f_{\ddot{F}\left(e_{i}\right)}\left(y_{k}\right)-f_{\ddot{G}\left(e_{i}\right)}\left(y_{k}\right)\right|
\end{array}\right] \\
=1-\frac{1}{8 n} \sum_{j=1}^{n}\left[\begin{array}{l}
\left|S_{\ddot{G}\left(e_{i}\right)}\left(x_{j}\right)-S_{\ddot{F}\left(e_{i}\right)}\left(x_{j}\right)\right| \\
+\left|t_{\ddot{G}\left(e_{i}\right)}\left(x_{j}\right)-t_{\ddot{F}\left(e_{i}\right)}\left(x_{j}\right)\right| \\
+\left|f_{\ddot{G}\left(e_{i}\right)}\left(x_{j}\right)-f_{\ddot{F}\left(e_{i}\right)}\left(x_{j}\right)\right|
\end{array}\right]
\end{gathered}
$$

$$
-\frac{1}{8 p} \sum_{k=1}^{p}\left[\begin{array}{c}
\left|S_{\ddot{G}\left(e_{i}\right)}\left(y_{k}\right)-S_{\ddot{F}\left(e_{i}\right)}\left(y_{k}\right)\right| \\
+\left|t_{\ddot{G}\left(e_{i}\right)}\left(y_{k}\right)-t_{\ddot{F}\left(e_{i}\right)}\left(y_{k}\right)\right| \\
+\left|f_{\ddot{G}\left(e_{i}\right)}\left(y_{k}\right)-f_{\ddot{F}\left(e_{i}\right)}\left(y_{k}\right)\right|
\end{array}\right]
$$$$
=\mathrm{M}((\ddot{G}, \mathrm{~B}),(\ddot{F}, \mathrm{~A}))
$$

$\left(\mathrm{C}_{2}\right)\left|S_{\ddot{F}\left(e_{i}\right)}\left(x_{j}\right)-S_{\ddot{G}\left(e_{i}\right)}\left(x_{j}\right)\right| \leq 2$,

$$
\left|t_{\ddot{F}\left(e_{i}\right)}\left(x_{j}\right)-t_{\ddot{G}\left(e_{i}\right)}\left(x_{j}\right)\right| \leq 1,
$$$$
\left|f_{\ddot{F}\left(e_{i}\right)}\left(x_{j}\right)-f_{\ddot{G}\left(e_{i}\right)}\left(x_{j}\right)\right| \leq 1
$$$$
\text { and }
$$

$\left|S_{\ddot{F}\left(e_{i}\right)}\left(y_{k}\right)-S_{\ddot{G}\left(e_{i}\right)}\left(y_{k}\right)\right| \leq 2$,

$\left|t_{\ddot{F}\left(e_{i}\right)}\left(y_{k}\right)-t_{\ddot{G}\left(e_{i}\right)}\left(y_{k}\right)\right| \leq 1$,

$\left|f_{\ddot{F}\left(e_{i}\right)}\left(y_{k}\right)-f_{\ddot{G}\left(e_{i}\right)}\left(y_{k}\right)\right| \leq 1$

So using formula

$M_{e_{i}}((\ddot{F}, \mathrm{~A}),(\ddot{G}, \mathrm{~B})) \in[0,1] \Rightarrow M((\ddot{G}, \mathrm{~B}),(\ddot{F}, \mathrm{~A})) \in[0,1]$

$\left(\mathrm{C}_{3}\right) \mathrm{M}((\ddot{F}, \mathrm{~A}),(\ddot{G}, \mathrm{~B}))=1$

$$
\begin{aligned}
& \Leftrightarrow \frac{1}{\operatorname{Card}(A \cup B)} \sum_{e_{i} \in(A \cap B)} M_{e_{i}}((\ddot{F}, \mathrm{~A}),(\ddot{G}, \mathrm{~B}))=1 \\
& \Leftrightarrow M_{e_{i}}((\ddot{F}, \mathrm{~A}),(\ddot{G}, \mathrm{~B}))=1 ; \forall e_{i} \in(A \cap B) \\
& \Leftrightarrow \frac{1}{8 n} \sum_{j=1}^{n}\left[\begin{array}{c}
\left|S_{\ddot{G}\left(e_{i}\right)}\left(x_{j}\right)-S_{\ddot{F}\left(e_{i}\right)}\left(x_{j}\right)\right| \\
+\left|t_{\ddot{G}\left(e_{i}\right)}\left(x_{j}\right)-t_{\ddot{F}\left(e_{i}\right)}\left(x_{j}\right)\right| \\
+\left|f_{\ddot{G}\left(e_{i}\right)}\left(x_{j}\right)-f_{\ddot{F}\left(e_{i}\right)}\left(x_{j}\right)\right|
\end{array}\right] \\
& +\frac{1}{8 p} \sum_{k=1}^{p}\left[\begin{array}{c}
\left|S_{\ddot{G}\left(e_{i}\right)}\left(y_{k}\right)-S_{\ddot{F}\left(e_{i}\right)}\left(y_{k}\right)\right| \\
+\left|t_{\ddot{G}\left(e_{i}\right)}\left(y_{k}\right)-t_{\ddot{F}\left(e_{i}\right)}\left(y_{k}\right)\right| \\
+\left|f_{\ddot{G}\left(e_{i}\right)}\left(y_{k}\right)-f_{\ddot{F}\left(e_{i}\right)}\left(y_{k}\right)\right|
\end{array}\right]=0 \\
& \Leftrightarrow\left|S_{\ddot{F}\left(e_{i}\right)}\left(x_{j}\right)-S_{\ddot{G}\left(e_{i}\right)}\left(x_{j}\right)\right|=0 \\
& \left|t_{\ddot{F}\left(e_{i}\right)}\left(x_{j}\right)-t_{\ddot{G}\left(e_{i}\right)}\left(x_{j}\right)\right|=0, \\
& \left|f_{\ddot{F}\left(e_{i}\right)}\left(x_{j}\right)-f_{\ddot{G}\left(e_{i}\right)}\left(x_{j}\right)\right|=0 \\
& \text { and } \\
& \left|S_{\ddot{F}\left(e_{i}\right)}\left(y_{k}\right)-S_{\ddot{G}\left(e_{i}\right)}\left(y_{k}\right)\right|=0, \\
& \left|t_{\ddot{F}\left(e_{i}\right)}\left(y_{k}\right)-t_{\ddot{G}\left(e_{i}\right)}\left(y_{k}\right)\right|=0 \text {, } \\
& \left|f_{\ddot{F}\left(e_{i}\right)}\left(y_{k}\right)-f_{\ddot{G}\left(e_{i}\right)}\left(y_{k}\right)\right|=0
\end{aligned}
$$

$\forall e_{i} \in \mathrm{A} \cap \mathrm{B}, \forall x_{j} \in \mathrm{U}_{1}$ and $\forall e_{i} \in(A \cap B), \forall y_{k} \in \mathrm{U}_{2}$

$$
\Leftrightarrow(\ddot{F}, \mathrm{~A})=(\ddot{G}, \mathrm{~B})
$$

$\left(\mathrm{C}_{4}\right) \mathrm{M}((\ddot{F}, \mathrm{~A}),(\ddot{G}, \mathrm{~B}))=0$

$$
\begin{aligned}
& \Leftrightarrow \frac{1}{\operatorname{Card}(A \cup B)} \sum_{e_{i} \in(A \cap B)} M_{e_{i}}((\ddot{F}, \mathrm{~A}),(\ddot{G}, \mathrm{~B}))=0 \\
& \Leftrightarrow M_{e_{i}}((\ddot{F}, A),(\ddot{G}, B))=0 \\
& \Leftrightarrow \frac{1}{8 n} \sum_{j=1}^{n}\left[\begin{array}{c}
\left|S_{\ddot{G}\left(e_{i}\right)}\left(x_{j}\right)-S_{\ddot{F}\left(e_{i}\right)}\left(x_{j}\right)\right| \\
+\left|t_{\ddot{G}\left(e_{i}\right)}\left(x_{j}\right)-t_{\ddot{F}\left(e_{i}\right)}\left(x_{j}\right)\right| \\
+\left|f_{\ddot{G}\left(e_{i}\right)}\left(x_{j}\right)-f_{\ddot{F}\left(e_{i}\right)}\left(x_{j}\right)\right|
\end{array}\right] \\
& +\frac{1}{8 p} \sum_{k=1}^{p}\left[\begin{array}{c}
\left|S_{\ddot{G}\left(e_{i}\right)}\left(y_{k}\right)-S_{\ddot{F}\left(e_{i}\right)}\left(y_{k}\right)\right| \\
+\left|t_{\ddot{G}\left(e_{i}\right)}\left(y_{k}\right)-t_{\ddot{F}\left(e_{i}\right)}\left(y_{k}\right)\right| \\
+\left|f_{\ddot{G}\left(e_{i}\right)}\left(y_{k}\right)-f_{\ddot{F}\left(e_{i}\right)}\left(y_{k}\right)\right|
\end{array}\right]=1 \\
& \Leftrightarrow\left|S_{\ddot{G}\left(e_{i}\right)}\left(x_{j}\right)-S_{\ddot{F}\left(e_{i}\right)}\left(x_{j}\right)\right|=2, \\
& \left|t_{\ddot{G}\left(e_{i}\right)}\left(x_{j}\right)-t_{\ddot{F}\left(e_{i}\right)}\left(x_{j}\right)\right|=1,
\end{aligned}
$$




\section{Available online at www.ijrat.org}

$$
\begin{aligned}
&\left|f_{\ddot{G}\left(e_{i}\right)}\left(x_{j}\right)-f_{\ddot{F}\left(e_{i}\right)}\left(x_{j}\right)\right|=1, \\
& \forall e_{i} \in(\mathrm{A} \cap \mathrm{B}), \forall x_{j} \in \mathrm{U}_{1} \\
&\left|S_{\ddot{F}\left(e_{i}\right)}\left(y_{k}\right)-S_{\ddot{G}\left(e_{i}\right)}\left(y_{k}\right)\right|=2, \\
&\left|t_{\ddot{G}\left(e_{i}\right)}\left(x_{j}\right)-t_{\ddot{F}\left(e_{i}\right)}\left(x_{j}\right)\right|=1, \\
&\left|f_{\ddot{G}\left(e_{i}\right)}\left(y_{k}\right)-f_{\ddot{F}\left(e_{i}\right)}\left(y_{k}\right)\right|=1, \\
& \forall e_{i} \in(\mathrm{A} \cap \mathrm{B}), \forall y_{k} \in \mathrm{U}_{2}
\end{aligned}
$$

$$
\begin{aligned}
\Leftrightarrow t_{\ddot{F}\left(e_{i}\right)}\left(x_{j}\right)=0, t_{\ddot{G}\left(e_{i}\right)}\left(x_{j}\right)=1 & \text { or } \\
t_{\ddot{F}\left(e_{i}\right)}\left(x_{j}\right)= & 1, t_{\ddot{G}\left(e_{i}\right)}\left(x_{j}\right)=0 \\
& \text { and } \\
f_{\ddot{F}\left(e_{i}\right)}\left(x_{j}\right)= & 0, f_{\ddot{G}\left(e_{i}\right)}\left(x_{j}\right)=1 \\
& \text { or } \\
f_{\ddot{F}\left(e_{i}\right)}\left(x_{j}\right)= & 1, f_{\ddot{G}\left(e_{i}\right)}\left(x_{j}\right)=0 ;
\end{aligned}
$$$$
\&
$$

$$
\begin{aligned}
& t_{\ddot{F}\left(e_{i}\right)}\left(y_{k}\right)=0, t_{\ddot{G}\left(e_{i}\right)}\left(y_{k}\right)=1 \\
& \text { or } \\
& t_{\ddot{F}\left(e_{i}\right)}\left(y_{k}\right)=1, t_{\ddot{G}\left(e_{i}\right)}\left(y_{k}\right)=0 ; \\
& \text { and } \\
& f_{\ddot{F}\left(e_{i}\right)}\left(y_{k}\right)=0, f_{\ddot{G}\left(e_{i}\right)}\left(y_{k}\right)=1 \\
& \text { or } \\
& f_{\ddot{F}\left(e_{i}\right)}\left(y_{k}\right)=1, f_{\ddot{G}\left(e_{i}\right)}\left(y_{k}\right)=0
\end{aligned}
$$

$$
\begin{gathered}
\left(\mathrm{C}_{5}\right)(\mathrm{F}, \mathrm{A}) \subseteq(\mathrm{G}, \mathrm{B}) \subseteq(\mathrm{P}, \mathrm{C}) \\
\Rightarrow t_{\ddot{F}\left(e_{i}\right)}\left(x_{j}\right) \leq t_{\ddot{G}\left(e_{i}\right)}\left(x_{j}\right) \leq t_{\ddot{P}\left(e_{i}\right)}\left(x_{j}\right) ; \\
f_{\ddot{F}\left(e_{i}\right)}\left(x_{j}\right) \geq f_{\ddot{G}\left(e_{i}\right)}\left(x_{j}\right) \geq f_{\ddot{P}\left(e_{i}\right)}\left(x_{j}\right) \\
t_{\ddot{F}\left(e_{i}\right)}\left(y_{k}\right) \leq t_{\ddot{G}\left(e_{i}\right)}\left(y_{k}\right) \leq t_{\ddot{P}\left(e_{i}\right)}\left(y_{k}\right) ; \\
f_{\ddot{F}\left(e_{i}\right)}\left(y_{k}\right) \geq f_{\ddot{G}\left(e_{i}\right)}\left(y_{k}\right) \geq f_{\ddot{P}\left(e_{i}\right)}\left(y_{k}\right) \\
\Rightarrow\left|t_{\ddot{F}\left(e_{i}\right)}\left(x_{j}\right)-t_{\ddot{P}\left(e_{i}\right)}\left(x_{j}\right)\right| \\
\geq\left|t_{\ddot{F}\left(e_{i}\right)}\left(x_{j}\right)-t_{\ddot{G}\left(e_{i}\right)}\left(x_{j}\right)\right| ; \\
\left|f_{\ddot{F}\left(e_{i}\right)}\left(x_{j}\right)-f_{\ddot{P}\left(e_{i}\right)}\left(x_{j}\right)\right| \\
\geq\left|f_{\ddot{F}\left(e_{i}\right)}\left(x_{j}\right)-f_{\ddot{G}\left(e_{i}\right)}\left(x_{j}\right)\right| \\
\Rightarrow\left|S_{\ddot{F}\left(e_{i}\right)}\left(x_{j}\right)-S_{\ddot{P}\left(e_{i}\right)}\left(x_{j}\right)\right| \\
\geq\left|S_{\ddot{F}\left(e_{i}\right)}\left(x_{j}\right)-S_{\ddot{G}\left(e_{i}\right)}\left(x_{j}\right)\right| ;
\end{gathered}
$$

$\left|S_{\ddot{F}\left(e_{i}\right)}\left(y_{k}\right)-S_{\ddot{P}\left(e_{i}\right)}\left(y_{k}\right)\right| \geq\left|S_{\ddot{F}\left(e_{i}\right)}\left(y_{k}\right)-S_{\ddot{G}\left(e_{i}\right)}\left(y_{k}\right)\right|$

$\Rightarrow M_{e_{i}}((\ddot{F}, \mathrm{~A}),(\ddot{P}, \mathrm{C})) \leq M_{e_{i}}((\ddot{F}, \mathrm{~A}),(\ddot{G}, \mathrm{~B}))$

$\Rightarrow \mathrm{M}((\ddot{F}, \mathrm{~A}),(\ddot{P}, \mathrm{C})) \leq \mathrm{M}((\ddot{F}, \mathrm{~A}),(\ddot{G}, \mathrm{~B}))$.

Similarly it can be proved that,

$\mathrm{M}((\ddot{F}, \mathrm{~A}),(\ddot{P}, \mathrm{C})) \leq \mathrm{M}((\ddot{G}, \mathrm{~B}),(\ddot{P}, \mathrm{C}))$.

Hence $\mathrm{M}((\ddot{F}, \mathrm{~A}),(\ddot{P}, \mathrm{C})) \leq \min (\mathrm{M}((\ddot{F}, \mathrm{~A}),(\ddot{G}, \mathrm{~B}))$, $\mathrm{M}((\ddot{G}, \mathrm{~B}),(\ddot{P}, \mathrm{C})), \forall(\ddot{P}, \mathrm{C}) \in \operatorname{VSS}\left(\mathrm{U}_{1}\right) \times \operatorname{VSS}\left(\mathrm{U}_{2}\right)$

\section{Example 3. 3. (With fixed parameter set)}

Let $\mathrm{U}_{1}=\{\mathrm{a}, \mathrm{b}\}, \mathrm{U}_{2}=\{\mathrm{x}, \mathrm{y}\}$ be a common universe with a fixed set of parameters $\mathrm{E}=\left\{e_{1}, e_{2} e_{3}, e_{4}\right\}$ and $\mathrm{A}=\left\{e_{1}, e_{3}\right\}$. Let $(\mathrm{F}, \mathrm{A})$ and $(\mathrm{G}, \mathrm{A})$ be two vague binary soft sets defined as follows :
$(\ddot{F}, \mathrm{~A})=\left\{\begin{array}{c}\left(e_{1},\left\langle\frac{[0.1,0.4]}{a}, \frac{[0.3,0.6]}{b}\right\rangle,\left\langle\frac{[0.6,0.8]}{x}, \frac{[0.5,0.7]}{y}\right\rangle\right), \\ \left(e_{3},\left\langle\frac{[0.5,0.7]}{a}, \frac{[0.8,0.9]}{b}\right\rangle,\left\langle\frac{[0.7,0.8]}{x}, \frac{[0.3,0.5]}{y}\right\rangle\right)\end{array}\right\}$
$(\ddot{G}, \mathrm{~A})=\left\{\begin{array}{c}\left(e_{1},\left\langle\frac{[0.2,0.6]}{a}, \frac{[0.6,0.7]}{b}\right\rangle,\left\langle\frac{[0.3,0.7]}{x}, \frac{[0.6,0.7]}{y}\right\rangle\right), \\ \left(e_{3},\left\langle\frac{[0.4,0.7]}{a}, \frac{[0.8,0.9]}{b}\right\rangle,\left\langle\frac{[0.7,0.8]}{x}, \frac{[0.5,0.6]}{y}\right\rangle\right)\end{array}\right\}$.

Using the above method Similarity Measure of these sets is found as $\mathrm{M}((\ddot{F}, \mathrm{~A}),(\ddot{G}, \mathrm{~A}))=0.45 \in[0,1]$

\section{Example 3.4. (With different parameter set)}

Let $U_{1}=\{\mathrm{a}, \mathrm{b}\}, U_{2}=\{\mathrm{x}, \mathrm{y}\}$ be a common universe with a fixed set of parameters $\mathrm{E}=\left\{e_{1}, e_{2}\right\}$ and $\mathrm{A}=\left\{e_{1}, e_{3}, e_{4}\right\}, \mathrm{B}=\left\{e_{3}, e_{4}, e_{5}\right\}$. Let $(\ddot{F}, \mathrm{~A})$ and $(\ddot{G}, \mathrm{~B})$ be two VBSS's defined as follows:

$(\ddot{F}, \mathrm{~A})=\left\{\begin{array}{l}\left(e_{1},\left\langle\left[\frac{[0.2,0.4]}{a}, \frac{[0.1,0.5]}{b}\right\rangle,\left\langle\left[\frac{[0.5,0.6]}{x}, \frac{[0.4,0.8]}{y}\right\rangle\right),\right.\right. \\ \left(e_{3},\left\langle\frac{[0.5,0.9]}{a}, \frac{[0.4,0.8]}{b}\right\rangle,\left\langle\frac{[0.7,0.9]}{x}, \frac{[0.3,0.04]}{y}\right\rangle\right), \\ \left(e_{4},\left\langle\frac{[0.1,0.3]}{a}, \frac{[0.3,0.6]}{b}\right\rangle,\left\langle\frac{[0.2,0.4]}{x}, \frac{[0.6,0.7]}{y}\right\rangle\right)\end{array}\right\}$
$(\ddot{G}, \mathrm{~B})=\left\{\begin{array}{c}\left(e_{3},\left\langle\frac{[0.3,0.7]}{a}, \frac{[0.5,0.9]}{b}\right\rangle,\left\langle\frac{[0.2,0.9]}{x}, \frac{[0.4,0.8]}{y}\right\rangle\right), \\ \left(e_{4},\left\langle\frac{[0.2,0.8]}{a}, \frac{[0.1,0.5]}{b}\right\rangle,\left\langle\frac{[0.6,0.0]}{x}, \frac{[0.4,0.9]}{y}\right\rangle\right), \\ \left(e_{5},\left\langle\frac{[0.4,0.6]}{a}, \frac{[0.3,0.7]}{b}\right\rangle,\left\langle\frac{[0.6,0.7]}{x}, \frac{[0.2,0.9]}{y}\right\rangle\right)\end{array}\right\}$.

Using the above mentioned method similarity measure between these sets is $\mathrm{M}((\ddot{F}, \mathrm{~A}),(\ddot{G}, \mathrm{~B}))=0.26 \in[0,1]$

Remark 3.4.1. (Some Special Cases)

Case ( $i): A$ and $B$ are empty subsets of $E$

$\mathrm{M}((\ddot{F}, \emptyset),(\ddot{G}, \emptyset))=\frac{1}{\operatorname{Card} \emptyset} \sum_{e_{i \in \emptyset}} M_{e_{i}}((\ddot{F}, \emptyset),(\ddot{G}, \emptyset))=0$ Case (ii): $A$ and $B$ are non-empty subsets of $E$ $\mathrm{M}((\ddot{F}, A),(\ddot{G}, \mathrm{~B}))=$

$\frac{1}{\operatorname{Card}(A \cup B)} \sum_{e_{i \in(A \cap B)}} M_{e_{i}}((\ddot{F}, \mathrm{~A}),(\ddot{G}, \mathrm{~B}))$

Case(iii) : $A \& B$ coincides with full parameter set $E$ $\mathrm{M}((\ddot{F}, \mathrm{E}),(\ddot{G}, \mathrm{E}))=\frac{1}{\operatorname{Card}(E)} \sum_{e_{i}} M_{e_{i \in E}}((\ddot{F}, \mathrm{E}),(\ddot{G}, \mathrm{E}))$

Case (iv) : $A=\emptyset, B$ is a non-empty subset of $E$

$\mathrm{M}((\ddot{F}, \varnothing),(\ddot{G}, \mathrm{~B}))=\frac{1}{\operatorname{card}(B)} \sum_{e_{i \in \emptyset}} M_{e_{i}}((\ddot{F}, \emptyset),(\ddot{G}, \mathrm{~B}))=0$

Case (v) : $A$ is a non-empty subset of $E, B=\varnothing$ $\mathrm{M}((\ddot{F}, A),(\ddot{G}, \varnothing))=\frac{1}{\operatorname{Card}(A)} \sum_{e_{i \in \emptyset}} M_{e_{i}}((\ddot{F}, \mathrm{~A}),(\ddot{G}, \emptyset))=0$

Case (vi): $A$ is a non-empty subset of $E$ and $B=E$ $\mathrm{M}((\ddot{F}, A),(\ddot{G}, \mathrm{E}))=\frac{1}{\operatorname{Card}(E)} \sum_{e_{i \in A}} M_{e_{i}}((\ddot{F}, \mathrm{~A}),(\ddot{G}, \mathrm{E}))$

Case (vii): $A=E$ and $B$ is a non-empty subset of $E$ $\mathrm{M}((\ddot{F}, E),(\ddot{G}, \mathrm{~B}))=\frac{1}{\operatorname{Card}(E)} \sum_{e_{i \in B}} M_{e_{i}}((\ddot{F}, \mathrm{E}),(\ddot{G}, \mathrm{~B}))$

Remark 3.4.2.

$\mathrm{A}$ and $\mathrm{B}$ are both empty or any one of them takes empty value, similarity measure between these sets will be always zero

\section{WEIGHTED SIMILARITY MEASURES FOR VBSS's \\ Definition 4.1.}


Let $\mathrm{U}_{1}=\left\{\mathrm{x}_{1}, \mathrm{x}_{2}, \ldots, \mathrm{x}_{\mathrm{n}}\right\}, \mathrm{U}_{2}=\left\{\mathrm{y}_{1}, \mathrm{y}_{2}, \ldots, \mathrm{y}_{\mathrm{p}}\right\}$ be the common universal set and $\mathrm{E}=\left\{\mathrm{e}_{1}, \mathrm{e}_{2}, \ldots, \mathrm{e}_{\mathrm{m}}\right\}$ be the set of parameters. Hence $(\ddot{F}, \mathrm{~A})=\left\{\ddot{F}\left(e_{i}\right) / \mathrm{i}=1,2, . ., \mathrm{m}\right\}$ and $(\ddot{G}, \mathrm{~B})=\left\{\ddot{G}\left(e_{i}\right) / \mathrm{i}=1,2, \ldots, \mathrm{m}\right\}$ are two families of vague binary soft sets. Suppose the weight of the elements in the universe of discourse $U_{1}$ is $w_{j}$ and the weight of the elements in the universe of discourse $U_{2}$ is denoted by $\mathrm{w}_{\mathrm{k}}{ }^{*}$ where $\mathrm{w}_{\mathrm{j}} \in[0,1]$ and $\mathrm{w}_{\mathrm{k}}{ }^{*} \in[0,1]$. Degree of similarity between vague binary soft sets $(\ddot{F}, \mathrm{~A})$ and $(\ddot{G}, \mathrm{~B})$ can be written by the weighting function denoted by $\mathrm{W}((\ddot{F}, \mathrm{~A}),(\ddot{G}, \mathrm{~B}))$ and is given by

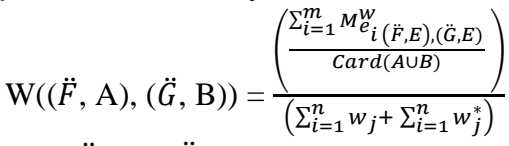

$M_{e_{i}}^{w}((\ddot{F}, \mathrm{~A}),(\ddot{G}, \mathrm{~B}))=$

$1-\frac{1}{8 n} \sum_{j=1}^{n} w_{j}\left[\begin{array}{c}\left|S_{\ddot{G}\left(e_{i}\right)}\left(x_{j}\right)-S_{\ddot{F}\left(e_{i}\right)}\left(x_{j}\right)\right| \\ +\left|t_{\ddot{G}\left(e_{i}\right)}\left(x_{j}\right)-t_{\ddot{F}\left(e_{i}\right)}\left(x_{j}\right)\right| \\ +\left|f_{\ddot{G}\left(e_{i}\right)}\left(x_{j}\right)-f_{\ddot{F}\left(e_{i}\right)}\left(x_{j}\right)\right|\end{array}\right]$

$-\frac{1}{8 p} \sum_{k=1}^{p} w_{k}^{*}\left[\begin{array}{c}\left|S_{\ddot{G}\left(e_{i}\right)}\left(y_{k}\right)-S_{\ddot{F}\left(e_{i}\right)}\left(y_{k}\right)\right| \\ +\left|t_{\ddot{G}\left(e_{i}\right)}\left(y_{k}\right)-t_{\ddot{F}\left(e_{i}\right)}\left(y_{k}\right)\right| \\ +\mid f_{\ddot{G}\left(e_{i}\right)}\left(y_{k)}-f_{\ddot{F}\left(e_{i}\right)}\left(y_{k}\right) \mid\right.\end{array}\right]$

\section{Example 4.1.1.}

Let $(\ddot{F}, \mathrm{~A})$ and $(\ddot{G}, \mathrm{~B})$ be two VBSS's defined as in example 3.3. Suppose that the weights allotted for $\mathrm{x}$, $\mathrm{y}, \mathrm{a}, \mathrm{b}$ are respectively $0.4,1.0,0.6,0.9$. Degree of similarity between the VBSS's $(\ddot{F}, \mathrm{~A})$ and $(\ddot{G}, \mathrm{~B})$ can be evaluated using the above mentioned formula. It got as $\mathrm{W}((\ddot{F}, \mathrm{~A}),(\ddot{G}, \mathrm{~B}))=0.3 \in[0,1]$

\section{CONCLUSION}

For fuzzy sets at present so many similarity measures are in use. But all the existing tools are found to get failed in the case of vague sets. Using a new tool-score function of vague values- Shyi-Ming Chen introduced similarity measure and weighted similarity measure for vague sets. In 2013 Chang Wang et.al., introduced similarity for vague soft sets. They also proved so many theorems connecting the three uncertainty tools similarity measure, distance measure, entropy of vague soft sets. Being one extension of fuzzy sets, vague sets play an important role in the application field like similarity measures. In this paper two similarity measures are developed for vague binary soft sets. The present result can be further extended to the other tools of uncertainty like entropy, distance measures etc and to its application field.

\section{REFERENCES}

[1] Athar Kharal, "Distance and Similarity Measures for soft sets", New Mathematics and Natural Computation. Vol.6, No.3, 321-334@World
Scientific Publishing Company, DOI : 10.1142/ S1793005710001724, 2010

[2] Chang Wang, Anjing Qu, "Entropy, Similarity measure and distance measure of vague soft sets and their relations", Information Sciences 244 92106, http://dx.doi.org/10.1016/j.ins.2013.05013, 2013

[3] Dan, Hu, Zhiyong Hong and Yong Wang, "A New Approach to Entropy and Similarity Measure of Vague soft sets". Hindawi Publishing Corporation, The Scientific World Journal, Volume 2014. Article ID610125.10pages, http://dx.doi.org/ 10. 1155 /2014 /61025, 2014

[4] Faxin Zhao and Z.M.Ma.Member IEEE, “ Similarity measures of vague sets based on the set-theoretic approach", IEEE International Conference on Systems, Man and Cybernetics, October 8-11, Taipei, Taiwan, 2006

[5] Dr.Francina Shalini.A and Remya.P.B, "Vague binary soft set and their properties", International journal of engineering, science and mathematics, http://www.ijesm.co.in, Email: ijesmj@gmail.com,Vol 7, Issue 1, Page 56-73, November (2018)

[6] Feng Sheng Xu, "A New Method on Similarity between Vague sets", 2009 World Congress on Computer Science and Information Engineering, 978-0-7695-3507-4/08 \$25.00@ 2008 IEEE, DOI; 10.1109/CSIE 2009. 27, IEEE Computer Society, 2008

[7] Jingli Lu, Xiaowei Yan, Dingrong Yuan, Zhangyan $\mathrm{Xu}$, "A new Similarity Measure for Vague sets", Feature Article, IEEE Intelligent Informatics Bulletin, Nove, Vol 6, No.2, November 2005

[8] Manash Jyoti Borah and Bipin Hazarika, "Some applications of similarity of soft sets", Journal of Intelligent \& Fuzzy Systems 33, DOI:10. 3233/JFS -17664, IOS Press , 3767-3777, 2017

[9] Pinaky Majumdar and S.K.Samanta, "On Similarity Measures of Fuzzy Soft Sets", Int.J.Advance. Soft Comput.Appl.,Vol.3, No.2, www.i-csrs.org, pp-1-8, July 2011

[10] Pinaki Majumdar and Syamal Kumar Samanta, "Decision making based on similarity measure of vague soft sets", Journal of Intelligent and Fuzzy Systems 24 , DOI : 10.3233/IFS-2012-0583 , 637646, 2013

[11] Qinrong Feng, Weinan Zheng, "New Similarity Measures of Fuzzy Soft Sets based on Distance Measures", Annals of Fuzzy Mathematics anf Informatics, ISSN: 2093-9310 (print version), ISSN :2287-6235(electronic version), http :// www. afmi. or.kr, Volume x, No.x (Month 201 y), pp.1-xx, 2013

[12] Shyi-Ming Chen, "Measures of Similarity between vague sets", Fuzzy Sets and Systems 74, 217-223, 1995

[13] Wenyi Zeng, Yibin Zhao, Yundong Gu , "Similarity measure for vague sets based on 
International Journal of Research in Advent Technology, Vol.7, No.4, April 2019

E-ISSN: 2321-9637

Available online at www.ijrat.org

implication functions", Knowledge based Systems, http:/ dx.doi.org /10.1016 /j.knosys . 2015.11.015, 0950-7051/@ 2015 published by Elsivier.B.V, 2015

[14] Zhicai Liu, Keyun Qin and Zheng Pei, Volume "Similarity Measure and Entropy of Fuzzy Soft Sets", Hindawi Publishing Corporation, The Scientific World Journal, http:// dx.doi.org/10. 1155 / 2014/161607, Article ID 161607, 10 pages, 2014 